\title{
Construcción del modelo SEIR que describe la evolución de la pandemia COVID - 19 en la región Tacna
}

\author{
Construction of the SEIR model that describes the evolution of the COVID \\ pandemic - 19 in the Tacna region \\ Construção do modelo SEIR que descreve a evolução da pandemia COVID - 19 na \\ região de Tacna \\ Jannina Quilca ${ }^{1}$ \\ Universidad Nacional del Altiplano, Perú
}

DOI: https://doi.org/10.35622/j.rg.2021.03.004

Recibido 08/07/2021/ Aceptado 20/07/2021

\begin{abstract}
RESUMEN. La aparición y posterior propagación de la COVID-19 ha generado el colapso de los sistemas sanitarios a nivel mundial, debido a la alta tasa de contagios y letalidad; evidenciadas en reportes oficiales diarios. Ante esta problemática la comunidad científica empieza a realizar estudios sobre la dinámica de la pandemia, mediante la construcción de modelos matemáticos a fin de dar a conocer la evolución de la pandemia y pronósticos a corto y mediano plazo. La presente investigación tiene por objetivo generalizar el modelo epidemiológico Susceptibles-Infectados-Recuperados (SIR) para construir un modelo matemático que describa la evolución de la pandemia COVID-19 en la región Tacna. Haciendo uso de los reportes oficiales emitidos por la Dirección Regional de Salud de la región entre abril y junio del 2021 se obtiene los parámetros de evolución, que representan las tasas de crecimiento y decrecimiento de un sistema de ecuaciones diferenciales ordinarias no lineales que constituyen el modelo matemático determinista SEIR. Las soluciones numéricas del modelo se obtienen utilizando herramientas computacionales. El desarrollo del modelo permite interpretar el avance de la pandemia y determinar el factor de contagio RO, contribuyendo a la toma de medidas de control epidemiológico adecuadas para frenar el número de contagios.
\end{abstract}

PALABRAS CLAVE: Covid-19, factor de contagio, SEIR, sistema de ecuaciones diferenciales.

ABSTRACT. The appearance and subsequent spread of COVID-19 have generated the collapse of health systems worldwide due to the high rate of infections and fatalities, evidenced in daily official reports. Faced with this problem, the scientific community begins to carry out studies on the dynamics of the pandemic through the construction of mathematical models to publicize the evolution of the pandemic and short and medium-term forecasts. This research aims to generalize the Susceptible-InfectedRecovered (SIR) epidemiological model to build a mathematical model that describes the

${ }^{1}$ Correspondencia: jaquilcac@est.unap.edu.pe 
evolution of the COVID-19 pandemic in the Tacna region. Using the official reports issued by the Regional Health Directorate of the area between April and June 2021, the evolution parameters are obtained, representing the growth and decrease rates of a system of non-linear ordinary differential equations that constitute the model. Deterministic mathematician SEIR. The numerical solutions of the model are obtained using computational tools. The development of the model makes it possible to interpret the progress of the pandemic and determine the contagion factor RO, contributing to the taking of adequate epidemiological control measures to stop the number of infections.

KEYWORDS: Covid-19, contagion factor, SEIR, system of differential equations.

RESUMO. O surgimento e posterior disseminação do COVID-19 gerou o colapso dos sistemas de saúde em todo o mundo, devido ao alto índice de infecções e fatalidades; evidenciado em relatórios oficiais diários. Diante desse problema, a comunidade científica passa a realizar estudos sobre a dinâmica da pandemia, por meio da construção de modelos matemáticos para divulgar a evolução da pandemia e previsões de curto e médio prazo. A presente pesquisa tem como objetivo generalizar o modelo epidemiológico Susceptible-Infected-Recovered (SIR) para construir um modelo matemático que descreva a evolução da pandemia COVID-19 na região de Tacna. A partir dos relatórios oficiais emitidos pela Direção Regional de Saúde da região entre abril e junho de 2021, são obtidos os parâmetros de evolução, que representam as taxas de crescimento e decréscimo de um sistema de equações diferenciais ordinárias não lineares que constituem o modelo. . As soluções numéricas do modelo são obtidas por meio de ferramentas computacionais. O desenvolvimento do modelo permite interpretar a evolução da pandemia e determinar o fator de contágio R0, contribuindo para a tomada de medidas de controle epidemiológico adequadas para conter o número de infecções.

PALABRAS CLAVE: Covid-19, fator de contágio, SEIR, sistema de equações diferenciais.

\section{INTRODUCCIÓN}

A finales del 2019 las autoridades de la República popular China informaron sobre diferentes casos de neumonía viral de causa desconocida en Wuhan, que no cedía ante los tratamientos convencionales. El 12 de enero del 2020 la Organización Mundial de la Salud (OMS), señala que las infecciones presentadas se debían a un nuevo sindrome respiratorio severo agudo al que denominaron COVID-19; la cantidad de personas contagiadas se incrementó exponencialmente en China y en países vecinos, el 24 de enero se reporta el primer caso en Europa, asimismo el 19 de enero se reportó el primer caso en el continente americano, específicamente en Estados Unidos y el 26 de febrero el ministerio de Salud de Brasil, reporta el primer caso de la enfermedad. El 11 de marzo del mismo año la OMS declara a la COVID-19 como pandemia debido al elevado número de contagios y muertos que se presentaron en 114 países. 
En Perú el primer caso de COVID 19 se reportó el 6 de marzo de 2020 y a partir del lunes 16 de marzo se implementaron medidas de aislamiento, que consistian en la suspensión de toda actividad de carácter público, privado y académico; restricciones que tuvieron rigor hasta el 1 de julio de 2020. Posteriormente, el gobierno implementó una cuarentena focalizada en las ciudades más afectadas por el virus, habilitando de manera paulatina los sectores laborales, pasando a lo que hoy se conoce como la "nueva normalidad", donde existen restricciones puntuales por niveles de contagio ya sea moderado, alto, muy alto o extremo. El primer caso confirmado de la Pandemia en Tacna, fue el 29 de marzo de 2020 y a la quincena de junio de 2021 el número de pacientes hospitalizados se ha duplicado y las camas en la Unidad de Cuidados Intensivos (UCI) se encuentran copadas. Se mantiene la preocupación por la expansión de la variante india en la región vecina de Arequipa.

La amenaza global que supone el virus del COVID-19 se debe a mecanismos no lineales de transmisión que derivan en un avance en el número de contagios (Pérez et al., 2020). Bajo esta premisa se hace necesario conocer el comportamiento reproductivo de la pandemia, a fin de contar con información precisa, confiable y oportuna que permita conocer el posible comportamiento de la enfermedad. Múltiples estudios se llevan a cabo en todo el mundo en la búsqueda de modelos de simulación que permitan conocer la dinámica de contagios y predecir parámetros de manera que se puedan anticipar los posibles escenarios de transmisión y por lo tanto contribuir con la toma de decisiones.

Los modelos empleados muestran variabilidad por las diferencias del comportamiento del COVID-19 en cada región, lo cual es explicado por las diferencias ambientales, genotipicas y culturales, además de las medidas tomadas por las autoridades regionales y locales.

El modelo base para enfermedades infecciosas se encarga del estudio de los Susceptibles, Infectados y Recuperados (SIR), siendo desarrollado por Kermack y Mc Kendrick en 1927, es representado por un sistema de ecuaciones diferenciales ordinarias no lineales, 
cuyas variables en estudio cambian a lo largo del tiempo. El modelo considera que la población es fija y que cada individuo sólo puede pertenecer a uno de los siguientes conjuntos: infectados, susceptibles o recuperados, además cuenta con dos parámetros: la tasa de transmisión de la enfermedad y la tasa de recuperación (Manrique et al., 2020). El modelo SIR puede ser extendido según la complejidad y singularidad de la pandemia, de manera que se estudien otros parámetros importantes que permitan describir adecuadamente la dinámica de contagios del Covid-19 en una población específica.

Actualmente la región Tacna se encuentra muy afectada por la pandemia COVID-19, debido al incremento en el número de contagios diarios, siendo necesario realizar estudios sobre la dinámica de contagios, de manera que se pueda tener información confiable acerca del nivel de propagación de la pandemia, así como el proceso de recuperación y fallecimiento de la población. Dado que la construcción de modelos matemáticos en epidemiologia permite describir y explicar el comportamiento de un patógeno (Vidal et al., 2020), la presente investigación aporta un modelo que ayuda en la toma de decisiones respecto a las medidas de control sanitario, siendo una gran contribución para comprender la dinámica de la pandemia COVID-19 que desde su aparición se convirtió en un problema de salud pública y viene generando el colapso de los sistemas sanitarios a nivel mundial.

Bajo este contexto se planteó como objetivo generalizar el modelo epidemiológico SIR para construir un modelo que describa la evolución de la pandemia COVID-19 en la región Tacna a partir de los datos oficiales emitidos por la DIRESA de la región. Se buscó además formular un sistema de ecuaciones diferenciales ordinarias que permita determinar el valor de los parámetros epidemiológicos de la pandemia COVID-19 a partir de los factores de evolución y el factor de contagio RO.

\subsection{Modelo SIR}


La modelización en epidemiologia se constituye en una herramienta para estudiar la propagación de enfermedades infecciosas emergentes y reemergentes, debido a que éstas presentan una dinámica compleja (Pliego, 2011). En su mayoría los modelos matemáticos se basan en el uso de ecuaciones diferenciales ordinarias, siendo de carácter continuo y determinista (Fresnadillo et al., 2013)

El modelo SIR propuesto por Kermack y McKendrick en 1927, separa a la población en tres categorias: Susceptibles (S), Infectados (I) y Removidos (R) (Floquet et al., 2021). El modelo SIR destaca porque permite simular la evolución de una enfermedad en tres escenarios básicos y se puede ajustar a problemas de contagios de enfermedades y en una determinada localidad.

Según Delgado (2021) las personas susceptibles son aquellas que pueden ser infectadas por un individuo contagiado. Los infectados son quienes se hallan con la enfermedad y pueden contagiar a otros y los recuperados son individuos que no se pueden infectar pues adquirieron inmunidad (temporal o permanente) y tampoco pueden infectar a otros porque se curaron de la enfermedad.

Figura 1: Flujo del modelo SIR básico
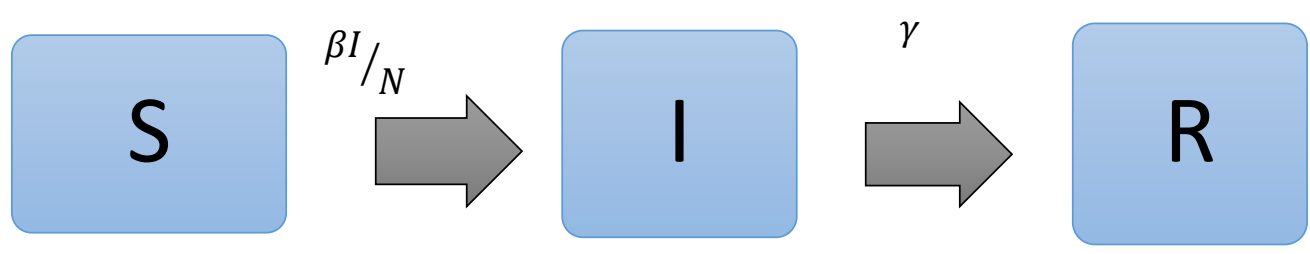

El modelo básico SIR asume que la población total se mantiene constante en el tiempo, es decir no considera los nacimientos ni defunciones, siendo la suma de los susceptibles, infectados y recuperados: $N=S(t)+I(t)+R(t)$

En este modelo se requiere conocer el valor de los parámetros $(\beta, \gamma)$ para establecer la dinámica de transmisión de contagios en una localidad determinada (Barroso, 2020). La probabilidad de infección 
o tasa de transmisión de la enfermedad $(\beta)$ se define como el producto del número de contactos por unidad de tiempo y la probabilidad de contagio e indica el número de contactos que son suficientes para que exista transmisión; en efecto, la expresión $\beta I / N$ indica el número de contactos infectados por unidad de tiempo de una persona susceptible, siendo $\beta I S / N$ el número de casos nuevos por unidad de tiempo. Por otra parte, la tasa de recuperación $(\gamma)$ indica la probabilidad por unidad de tiempo a la que los infectados se recuperan. El periodo medio de latencia (L) será entonces la inversa de la tasa de recuperación $L=1 / \gamma$.

Para transmitir la enfermedad, el individuo infeccioso tiene que estar en contacto con los susceptibles, un infeccioso transmite la enfermedad con una tasa de contagio $\beta$. Luego una fracción de infectados por unidad de tiempo deja esa clase para convertirse en recuperados. Lo que lleva al sistema de ecuaciones diferenciales del modelo SIR (Abelló et al., 2020).

Donde:

$$
\begin{gathered}
\frac{d S}{d t}=-\frac{\beta I}{N} S \\
\frac{d I}{d t}=\frac{\beta I}{N} S-\gamma I \\
\frac{d R}{d t}=\gamma I
\end{gathered}
$$

- $\beta$ : tasa de infección

- $\gamma$ : tasa de recuperación

- $\quad N$ : población total

Las condiciones iniciales del modelo son:

$$
S(0)=S_{0}=N-I_{0}, \quad I(0)=I_{0}, R(0)=0
$$

\subsection{Factor de Contagio $R_{0}$}

La tasa básica de reproducción es un parámetro muy importante puesto que permite describir la intensidad de una enfermedad infecciosa. González et al. (2021) lo define como una métrica utilizada 
para seguir la dinámica del virus, determinando así cuantas personas en promedio contagia cada infectado. Para saber si el número de infectados crecerá desatando una epidemia o decrecerá al punto de terminar extinguiéndose, el modelo SIR define su cálculo a partir de la siguiente ecuación.

$$
R_{0}=\frac{\beta}{\gamma}
$$

El uso del número reproductivo básico $\left(R_{0}\right)$ permite saber la probabilidad de trasmisión de una enfermedad. Si el parámetro toma un valor inferior a 1, la enfermedad culminara rápidamente; sin embargo, si su valor es superior a 1 se traduce en que la enfermedad pasara a ser una epidemia (Vidal et al., 2020). Si $R_{0}>1$ se desataría un brote epidémico con crecimiento exponencial, la situación expuesta se ilustra en la figura 2 , donde se supone un valor de $R_{0}=$ 3 , se observa que en un primer momento un individuo infectado contagia a 3 personas, quienes una vez infectados en un segundo momento contagiaran cada uno a otras 3 personas, teniendo ya en el tercer momento 9 infectados, si la situación continua el nivel de contagio será incontrolable en algún momento.

Figura 2: Dinámica de transmisión de una persona enferma para $\mathrm{RO}=3$

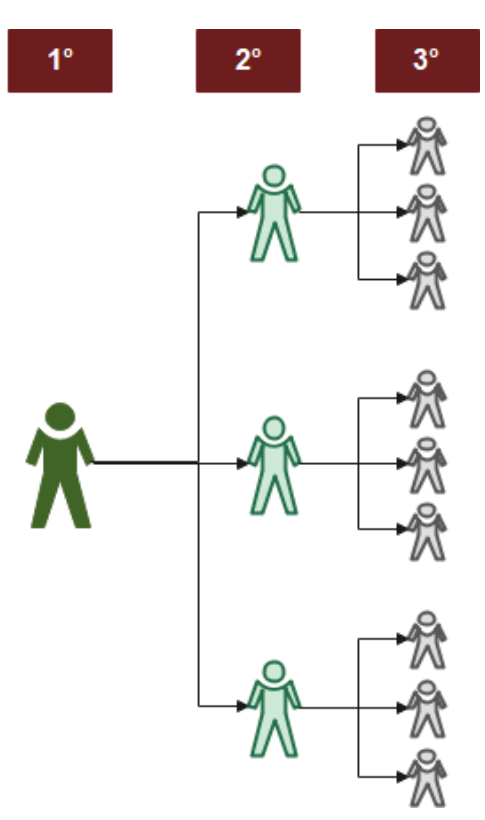

Para el caso del COVID-19, varios países realizaron la estimación del parámetro $R_{0}$, por ejemplo el Imperial College de Reino Unido 
encontró que el $R_{0}$ varía entre 1.5 y 3.5, siendo ese rango el más utilizado por las simulaciones realizadas a nivel internacional (Vidal et al., 2020). Un estudio más reciente realizado por Velasco (2021) calcula el número reproductivo básico encontrando que su valor asciende a 1.84 para el modelo SIR y 2.3 para el modelo SEIR. E1 cálculo del parámetro $R_{0}$ debe realizarse de manera cuidadosa; pues una diferencia mínima en su valor puede cambiar significativamente la dinámica de las curvas (Velasco, 2021). Motivo por el cual la presente investigación se utiliza el valor de 2.3 , basado en la revisión bibliográfica previa.

Por otra parte el número de reproducción efectivo $\left(R_{e}\right)$, mide el promedio de personas que pueden contagiarse en un momento del tiempo, representando así el valor del umbral que la enfermedad (García, 2020). Si $R_{e}<1$, la enfermedad se extingue pues I(t) decrece monótonamente tendiendo a cero para $t \rightarrow+\infty$. Por otra parte, si $R_{e}>$ 1 entonces $\mathrm{I}(\mathrm{t})$ crecerá produciendo una epidemia, una vez alcanzado su valor máximo (pico de la enfermedad) procederá a decrecer.

\section{MÉTODO}

Para construir un modelo a partir de la generalización del modelo epidemiológico SIR, se aplica el método inductivo. El acoplamiento de un compartimento adicional al modelo base SIR, permite definir un sistema de ecuaciones diferenciales ordinarias de tipo SEIR, que considera a los susceptibles, expuestos, infectados y recuperados (Birbuet \& López, 2020).

El estudio se encuentra enmarcado dentro del enfoque cualitativo. Es de carácter longitudinal, dado que permite conocer la evolución de la pandemia COVID-19 a través del tiempo. Los datos utilizados para la estimar los parámetros y construir el modelo fueron obtenidos de los reportes diarios emitidos por la DIRESA Tacna. Para la simulación del modelo se consideró la población total de la región, reportada en el último censo de población y vivienda realizado por el Instituto Nacional de Estadística e Informática. 
Para aproximar la solución del sistema de ecuaciones diferenciales ordinarias del modelo SEIR se realizó la simulación en el software Matlab. Los parámetros del modelo fueron calculados a partir del valor del factor de contagio R0 y de las ecuaciones del modelo.

\section{RESULTADOS Y DISCUSIONES}

Para describir la evolución de la pandemia COVID-19 en la región Tacna se generalizó el modelo SIR, incluyendo más compartimentos de estudio y por consiguiente acoplando variables y parámetros. Se propone un modelo de tipo SEIR (Susceptibles, Expuestos, Infectados y Recuperados) que adiciona el compartimento de las personas que habiendo estado en contacto con un enfermo se infectan y pasan a un periodo de latencia (donde no presentan sintomas) para transformarse en infectados.

Figura 3: Flujo del modelo SEIR

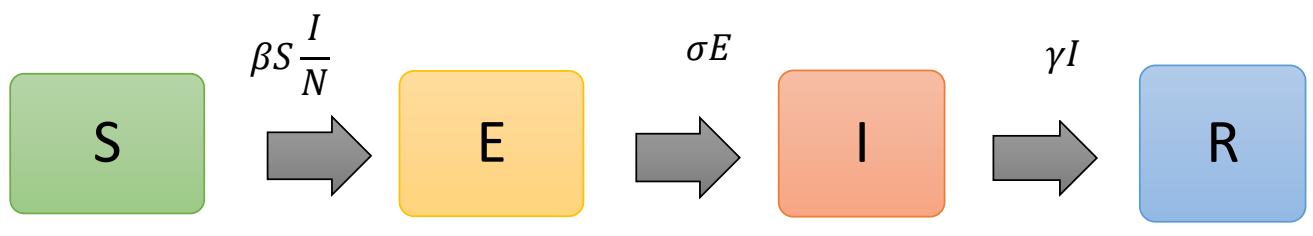

Según Culqui et al. (2020) la dinámica descrita por las ecuaciones del modelo SEIR:

- En un primer momento las personas de una población especifican están sanas y son Susceptibles a contagiarse.

- Una vez introducida la enfermedad los Susceptibles que se hallan en contacto con los enfermos se encuentran expuestos al virus.

- Los que estuvieron expuestos desarrollan la enfermedad, aumentando así el número de personas infectadas.

- De acuerdo con aspectos propios de cada individuo, una vez que se hayan infectado pueden recuperarse o en su defecto fallecer. 
- En algún momento la cantidad de personas recuperadas será mayor que las personas que aún no enfermaron.

- Por último, el brote epidemiológico se frenará cuando los susceptibles sean muy pocos y no se pueda propagar la enfermedad.

El modelo SEIR planteado consta de cuatro ecuaciones que miden la evolución de la población que se halla en cada compartimento a lo largo del tiempo. Además, considera que la población es constante en todo momento:

$$
\begin{aligned}
N & =S+E+I+R \\
\frac{d S}{d t} & =-\frac{\beta I}{N} S \\
\frac{d E}{d t} & =\frac{\beta I}{N} S-\sigma E \\
\frac{d I}{d t} & =\sigma E-\gamma I \\
\frac{d R}{d t} & =\gamma I
\end{aligned}
$$

Donde:

- $\beta$ : probabilidad de infección

- $\sigma$ : probabilidad de pasar de latente a infectado

- $\quad \gamma$ : probabilidad de recuperación

- $\quad N$ : población total

Tabla 1: Parámetros asociados al modelo SEIR

\begin{tabular}{lll}
\hline \multicolumn{1}{c}{ Parámetro } & \multicolumn{1}{c}{ Definición } & \multicolumn{1}{c}{ Fórmula } \\
\hline \multirow{3}{*}{ Tasa de } & & \multicolumn{1}{c}{$\beta=(\mathrm{tc})(\mathrm{ri})$} \\
transmisión & Probabilidad de que una persona & tc $=$ tasa de contacto \\
& susceptible sea infectada. & ri \\
& & $=$ riesgo de infeccion \\
\hline
\end{tabular}


Tasa de incubación

Tasa de recuperación

Periodo de transmisión

Periodo de incubación

Periodo de recuperación

Factor de contagio

Tasa de reproducción efectiva
Periodo de incubación de la enfermedad (desde que se infectó hasta que desarrolla sintomas)

Tiempo que dura la enfermedad.

Tempo en que las personas pasan de susceptibles a expuestos.

Tiempo trascurrido entre el comienzo de la infección y el $p e=\frac{1}{\sigma}$ momento en que se vuelve infeccioso.

$$
e=\frac{1}{\gamma}
$$
capaz de transmitir la enfermedad.

$$
R_{0}=\frac{\beta}{\gamma}
$$
enfermedad

$$
R_{e}=R_{0} \frac{S}{N}
$$

Fuente: elaboración propia

Los datos utilizados para la estimación de los parámetros y construcción del modelo se muestran en la Anexo 1 y fueron obtenidos de los reportes diarios emitidos por la DIRESA Tacna por un periodo de 91 dias, entre los meses de abril y junio del 2021.

Figura 4: Reporte de infectados DIRESA-Tacna abril-junio 2021 
Casos COVID-19 región Tacna

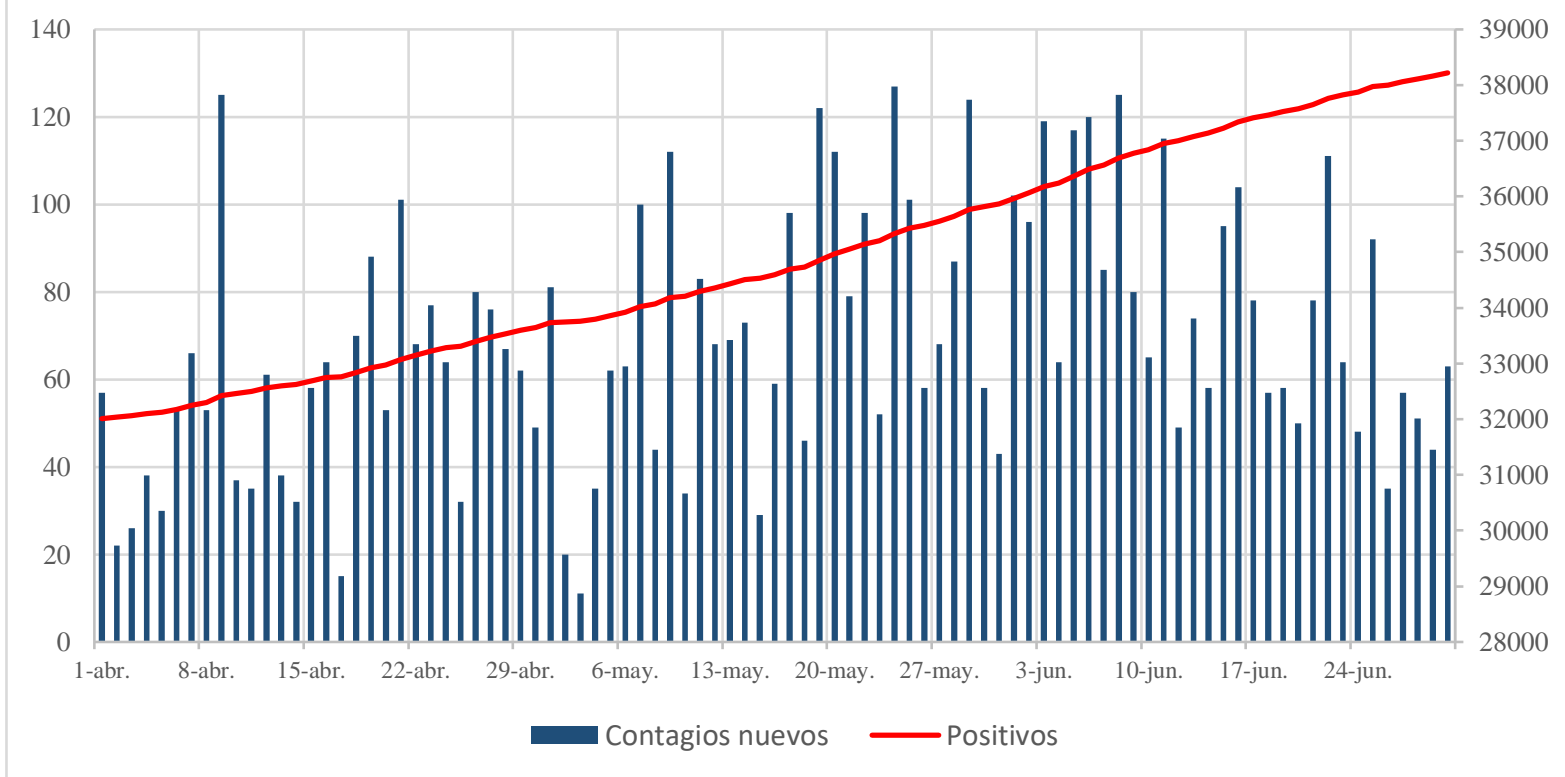

Los datos de infecciones reportados por la DIRESA Tacna, entre los meses de abril y junio que se muestran en la figura 4, permiten apreciar que los casos nuevos varian drásticamente entre un día y otro, dicha evolución genera variabilidad en los parámetros.

Para la simulación del modelo se consideran a las personas contagiadas en general, incluyendo los hospitalizados y personas en camas UCI en el compartimento de individuos infectados, por otra parte, los que recibieron alta médica y los que perdieron la vida se incluyen en el compartimento de recuperados. Dado que el modelo considera que la población total es constante, para efectos de estudio se considera el reporte oficial del Censo de población y vivienda 2017 realizado por el Instituto Nacional de estadística e Informática, el cual indica que la población total de Tacna asciende a 329332 personas.

Por otra parte, para estimar la tasa de recuperación se utiliza la ecuación 3 del modelo planteado conjuntamente con los datos emitidos por la DIRESA Tacna, los demás parámetros del modelo se calculan con las fórmulas mostradas en la tabla 1. 
Para describir la evolución de la COVID-19 en Tacna a partir del modelo SEIR planteado, se utiliza el software Matlab. Se consideran las siguientes condiciones iniciales:

$$
S(0)=297300, \quad E(0)=22, \quad I(0)=812, \quad R(0)=31198
$$

Los parámetros utilizados para la simulación del modelo, se definen de la siguiente manera:

- Factor de contagio $R_{0}=2.3$

- Porcentaje de población infectada en el día cero: $\frac{I(0)}{N}=0.0023$

- Periodo medio de incubación: pe $=5.6$ días

- Tasa de incubación: $\sigma=\frac{1}{5.6}=0.1786$

- Probabilidad de recuperación: $\gamma=0.1302$

- Probabilidad de infección: $\beta=0.2995$

A partir de pestos valores se realizó la simulación, cuya gráfica se presente en la figura 5. Ésta muestra la evolución de la pandemia en el número de individuos susceptibles (S), expuestos (E), infectados (I) y recuperados $(R)$ durante los meses de abril, mayo y junio en la región Tacna.

Figura 5: Simulación modelo SEIR para Tacna abril-junio 2021 


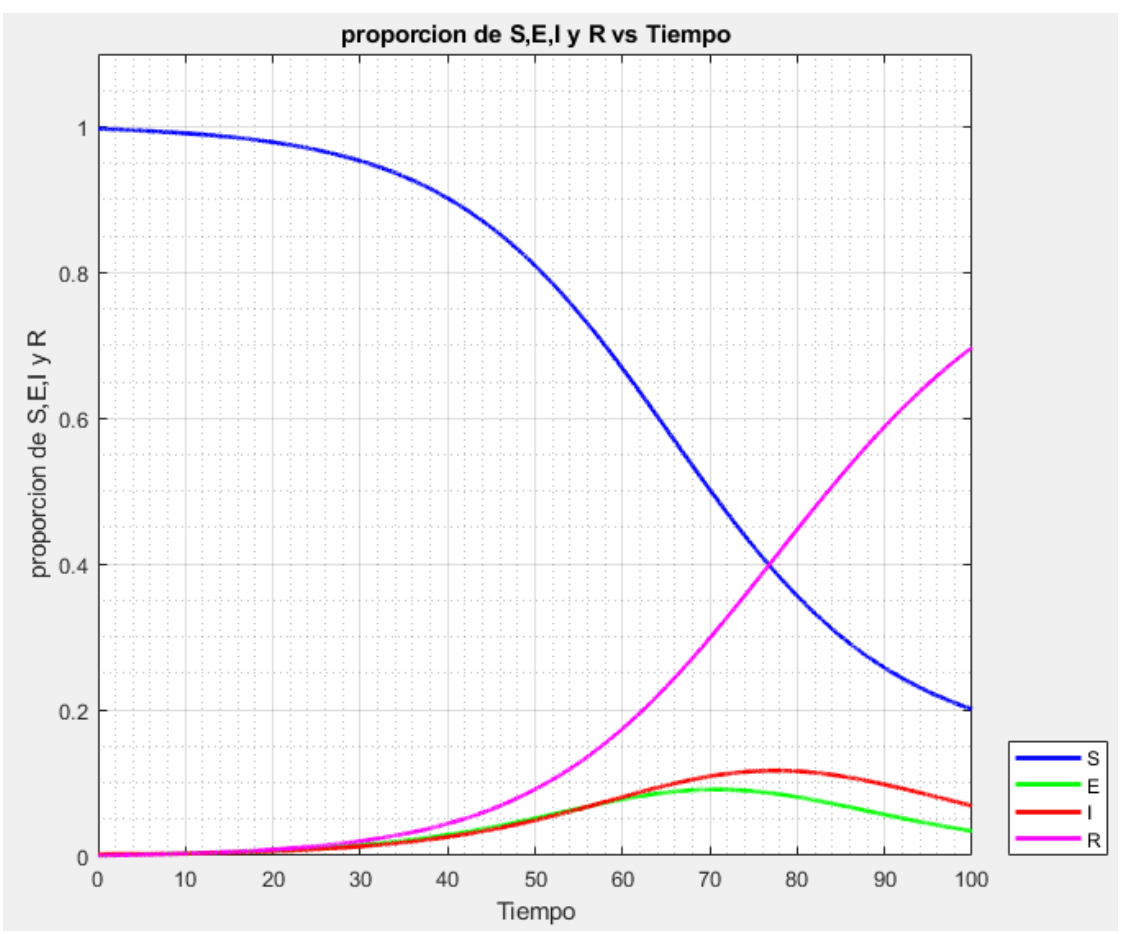

El comportamiento mostrado por las curvas es el esperado, puesto que los susceptibles, representados por la línea azul, decrecen en el tiempo a medida que se generan nuevos contagios. Los individuos infectados, expuestos y recuperados muestran una tendencia creciente, se encuentra además que el pico de infecciones se da el día 77 de la simulación (16 de junio) donde se registra 692 infectados, en el acumulado asciende a 37334 personas representando un $11,34 \%$ de la población. Por otra parte, durante el tiempo de simulación no se observa una estabilización en el comportamiento de las curvas, por lo que se puede inferir que la pandemia aún no se ha podido controlar.

\section{CONCLUSIONES}

Los modelos basados en ecuaciones diferenciales son utilizados para representar fenómenos de evolución, dinámica y pronóstico. Este tipo de modelos sirve como base para la construcción del modelo SEIR que permite describir la dinámica de la pandemia COVID 19 en la región Tacna. 
Los parámetros de evolución son estimados en base al reporte oficial de datos emitidos por la Dirección Regional de Salud de Tacna y el análisis de las ecuaciones diferenciales ordinarias planteadas en el modelo SEIR, utilizando Microsoft Excel.

El modelo SEIR estima la evolución de los individuos susceptibles, expuesto, infectados y recuperados, brinda pronostico a corto plazo debido a que el contagio es exponencial y es necesario actualizar constantemente los parámetros de evolución con los que se puede calibrar el modelo.

Se recomienda desarrollar modelos matemáticos para diversas regiones, en los cuales se pueda contemplar situaciones cada vez más conocidas de la pandemia, como el tiempo de inmunidad generada por la enfermedad, la vacunación, posibles confinamientos, la apertura de colegios, la permanencia en UCI, entre otros múltiples escenarios que se puedan presentar.

\section{REFERENCIAS}

Abelló, I., Guinovart, R., \& Morales, W. (2020). El modelo SIR básico y politicas antiepidémicas de salud pública para la COVID-19 en Cuba. Revista Cubana de Salud Pública, 46(0), Article 0. http://www.revsaludpublica.sld.cu/index.php/spu/article/vie $\mathrm{w} / 2597$

Barroso, V. (2020, abril 6). Una breve introducción al modelo SIR aplicado al caso del Covid-19. Instituto Complutense de Estudios Internacionales https://www.ucm.es/icei/file/iceipapercovid12

(ICEI).

Birbuet, J., \& López, R. (2020, abril 18). Dinámica de expansión de

la Covid-19 en Bolivia durante las primeras seis semanas.

Guardiana. Recuperado de http://bit.ly/pdf_covid_bol

Culqui, M., Nasimba, J., \& Chiliquinga, E. (2020). Aplicación del modelo matemático SEIR en la pandemia por Covid-19, relevancia en salud pública. Revista Vive, 3(9), 275-289. https://doi.org/10.33996/revistavive.v3i9.66

Delgado, J. (2021). Modelo dinámico de la pandemia de COVID19. Sanidad Militar, 77(1), 7-16. https://doi.org/10.4321/s188785712021000100002 
Floquet, S., Nobre, T., Pantaleão, R., Der Laan, A., Serafim, P, Pereira, P., Magalhães, T., \& da Silva, J. (2021). A matemática no combate a epidemia: estudo sobre a covid-19 na região do vale do são Francisco. Revista de Educação da Universidade Federal do Vale do São Francisco, 11(24), 100-146.

Fresnadillo, M., García, E., Martín, A., \& Garcia, J. (2013). Modelización matemática de la propagación de enfermedades infecciosas: De dónde venimos y hacia dónde vamos. 26(2), 8191.

García, L. (2020). Modelos matemáticos compartimentales en epidemiologia.pdf.

González, O., Chamorro, J., \& Rivas, G. (2021). Planificación y Gestión Integral de la Pandemia COVID-19 en Paraguay: Importancia de la integración de herramientas analiticas epidemiológicas, económicas y sociales. www.desarrollo.edu.py. https://datos.conacyt.gov.py/proyectos/nid/3411

Manrique, F., Agudelo, C., González, V., Gutiérrez, O., Téllez, C., \& Herrera, G. (2020). Modelo SIR de la pandemia de COVID-19 en Colombia. Rev. salud pública, 22, 1-9.

Pérez, R., Curra, D., \& Almaguer, L. (2020). Análisis preliminar de modelos SIRD para la predicción de la COVID-19: Caso de la provincia de Holguin. 10(2). http://www.revistaccuba.cu/index.php/revacc/article/view/8 $24 / 838$

Pliego, E. (2011). Modelos Epidemiológicos de Enfermedades Virales Infecciosas. Universidad Autónoma de Puebla.

Velasco, J. (2021). Modelos epidemiológicos, demografía y $R O$. https://doi.org/ 10.13140/RG.2.2.29595.39202

Vidal, M., Baldoquín, W., Durán, F., Bermejo, P. (2020). Número Básico de Reproducción (R0). Educación Médica Superior, 34(4). http:/ / scielo.sld.cu/scielo.php?script=sci_abstract\&pid=S086 4-21412020000400018\&lng=es\&nrm=iso\&tlng=es

Vidal, M., Guinovart, R., Baldoquín, W., Valdivia, N., \& Morales, W. (2020, junio 1). Modelos matemáticos para el control epidemiológico.

http://scielo.sld.cu/scielo.php?script=sci_arttext\&pid=S086421412020000200026 